\title{
So funktioniert die Abrechnung der Kompressionsbehandlung
}

Bei vielen phlebologischen Erkrankungen ist neben der Bewegungstherapie die Kompressionsbehandlung von grundlegender Bedeutung. Im EBM gibt es mit der Nr. 02313 eine eigenständige Abrechnungsposition, die auch im hausärztlichen Bereich ohne den Nachweis einer Zusatzqualifikation berechnungsfähig ist. Dabei ist zu beachten, dass diese Leistung nur beim Vorliegen einer chronisch venösen Insuffizienz (ICDCode I87.2), einem postthrombotischen Syndrom (I87.0X), einer oberflächlichen und tiefen Beinvenenthrombose (ICD I80.X) oder einem Lymphödem (I89.0) berechnet werden kann.

\section{MMW-KOMMENTAR}

Obligater Leistungsinhalt der Nr. 02313 EBM ist die Kompressionstherapie und die Dokumentation des Beinumfangs an mindestens drei Messpunkten zu Beginn der Behandlung und danach alle vier Wochen. Die Leistung

\section{Tab. 2 Abrechnungsbeispiel:} 60-jährige Patientin mit postthrombotischem Syndrom beidseitig oder ähnlichen Erkrankungen

\begin{tabular}{|l|l|l|}
\hline EBM & Legende & Euro \\
\hline 03040 & Grundpauschale & 44,27 \\
\hline 03004 & Versichertenpauschale & \\
\hline 02320 & Chronikerpauschale & \\
\hline 03230 & $\begin{array}{l}\text { Problemorientiertes } \\
\text { ärztliches Gespräch }\end{array}$ & 9,24 \\
\hline $\begin{array}{l}30401 \\
\times 2\end{array}$ & $\begin{array}{l}\text { Intermittierende apparati- } \\
\text { ve Kompressionstherapie }\end{array}$ & 6,98 \\
\hline $\begin{array}{l}02313 \\
\times 2\end{array}$ & $\begin{array}{l}\text { Anschließende Kompres- } \\
\text { sionstherapie z. B. mit } \\
\text { Kompressionsbinden }\end{array}$ & 11,70 \\
\hline Summe & $\mathbf{7 2 , 1 9}$ \\
\hline
\end{tabular}

ist pro Sitzung und pro Bein abrechenbar und mit 57 Punkten, aktuell also mit 5,85 Euro bewertet. Die maximale Abrechnungsmenge ist auf 4.244 Punkte pro Quartal beschränkt, was 74 Anwendungen entspricht. Dieser Höchstwert ist allerdings auf den Arztfall bezogen. In einer Gemeinschaftspraxis erhöht sich deshalb die Menge um die Anzahl der Ärzte.

Von der gleichzeitigen Abrechnung ausgeschlossen sind die Nr. 02310 für die Behandlung einer sekundär heilenden Wunde und die Nr. 02311 für die Behandlung eines diabetischen Fußes. Die Nr. 02312 für die Behandlung eines Ulcus cruris ist zwar nicht explizit ausgeschlossen, allerdings wird in der Legende wie auch in jener der Nr. 02313 eine Kompressionstherapie obligat gefordert. Damit schließen sich die beiden Positionen de facto gegenseitig aus.

Beachtenswert ist hingegen, dass die apparative Kompressionstherapie nach Nr. 30401 berechnungsfähig ist. Für diese Leistung muss mindestens eine der folgenden Diagnosen vorliegen: Artherosklerose der Extremitätenarterien bei umschriebenem Ödem, Varizen der unteren Extremitäten mit Ulzeration, postthrombotisches Syndrom, chronisch venöse Insuffizienz, Lymphödem, Ulcus cruris venosum, progressive systemische Sklerose, sonstige näher bezeichnete angeborene Fehlbildungen des peripheren Gefäßsystems, hereditäres Lymphödem oder Folgen von Verletzungen der unteren Extremität mit umschriebenem Ödem. Man sieht, dass diese Aufzählung sich z. T. mit jener der Nr. 02313, siehe oben, überlappt. Da die apparative Kompression vor einer manuellen Kompressionsbehandlung medizinisch durchaus denkbar ist, bei der Nr. 30401 kein Höchstwert besteht und die beiden Leistungen nebeneinander nicht ausgeschlossen sind, ist eine gemeinsame Abrechnung je Sitzung und je Bein möglich. 